\title{
Ferroelectric effects in PZT
}

\author{
L. Bellaiche, J. Padilla and David Vanderbilt \\ Department of Physics and Astronomy, Rutgers University, Piscataway, New Jersey 08855-0849
}

\begin{abstract}
First-principles calculations are performed to investigate alloying and ferroelectric effects in lead zirconate titanate (PZT) with high Ti composition. We find that the main effect of alloying in the paraelectric phase of PZT is the existence of two sets of $\mathrm{B}-\mathrm{O}$ bonds, i.e., shorter $\mathrm{Ti}-\mathrm{O}$ bonds vs. longer $\mathrm{Zr}-\mathrm{O}$ bonds. On the other hand, ferroelectricity leads to the formation of very short covalent $\mathrm{Ti}-\mathrm{O}$ bonds and to the formation of covalent chains of $\mathrm{Pb}-\mathrm{O}$ bonds. The covalency in the ferroelectric phase is mainly induced by an enhancement of hybridization between Ti $3 d$ and $\mathrm{O} 2 p$, and between $\mathrm{Pb} 6 s$ and $\mathrm{O} 2 p$. These hybridizations induce a striking decrease of the effective charges when going from the paraelectric to the ferroelectric phase of PZT.
\end{abstract}

\section{INTRODUCTION}

The technologically important lead zirconate titanate alloy (i.e., $\mathrm{PbZr}_{1-x} \operatorname{Ti}_{x} \mathrm{O}_{3}$ usually denoted as PZT) has an interesting phase diagram [1]. Increasing the $\mathrm{Ti}$ $x$ composition yields progressively the following ground state phases: an antiferroelectric orthorhombic phase for $x \lesssim 0.1$, a ferroelectric rhombohedral $\mathrm{FE}_{2}$ phase for $0.1 \lesssim x \lesssim 0.4$, another ferroelectric rhombohedral $\mathrm{FE}_{1}$ phase for $0.4 \lesssim x \lesssim 0.5$, and finally a tetragonal ferroelectric phase for $x$ larger than $50 \%$. The high-temperature phase of this alloy at all compositions is the cubic perovskite structure.

Previous theoretical studies [2] focused on the long-range B-site ordering effects in PZT for a Ti composition equal to 0.5, i.e., close to the morphotropic phase boundary between rhombohedral and tetragonal ferroelectric phases. The subject of the present theoretical study is rather different: we will investigate alloying and ferroelectric effects on structural, chemical and dielectric properties in the tetragonal phase of the PZT alloy. In other words, we would like to know what are the effects of alloying and ferroelectricity on bond lengths, chemical bonding and Born effective charges in PZT with high Ti content.

\section{METHOD}

We focus on the ordered structure shown in Fig. 1 and exhibiting a Ti composition equal to $2 / 3$. The B-site ordering of this supercell consists of one $\mathrm{Zr}$ plane 
alternating with two Ti planes along the [001] direction. We perform local-density approximation (LDA) calculations on this supercell using the Vanderbilt ultrasoftpseudopotential scheme [3], and including the semicore shells for all the metals considered. Specifically, the $\mathrm{Pb} 5 d, 6 s$ and $6 p$, the $\mathrm{Zr} 4 s, 4 p, 4 d$ and $5 s$, the $\mathrm{Ti}$ $3 s, 3 p, 3 d$ and $4 s$, and the $\mathrm{O} 2 s$ and $2 p$ electrons are treated as valence electrons. We choose the plane-wave cutoff to be 25 Ry and use the Ceperley-Alder exchange and correlation [4] as parameterized by Perdew and Zunger [5]. The first-principles calculations throughout this work are performed using a $(6,6,2)$ Monkhorst-Pack mesh [6]. Further technical details of the procedure used in the present study can be found in Ref. [7].

In fact, we perform two different calculations corresponding to two different symmetries of the structure shown in Fig. 1: (1) using a centrosymmetric cell (i.e., exhibiting an inversion symmetry about the central $\mathrm{Pb}$ atom); and (2) using a ferroelectric cell (i.e., relaxing the inversion symmetry constraint). Results of calculation (1) identify the alloying effects on various physical properties, while comparison of (1) with (2) allows us to isolate the ferroelectric effects on those properties.

The lattice parameter, the axial ratio c/a and the atomic positions along the [001] (compositional) direction are optimized in calculation (1) by minimizing the total energy and the Hellmann-Feynman forces, the latter being converged to within 0.02 $\mathrm{eV} / \AA$.

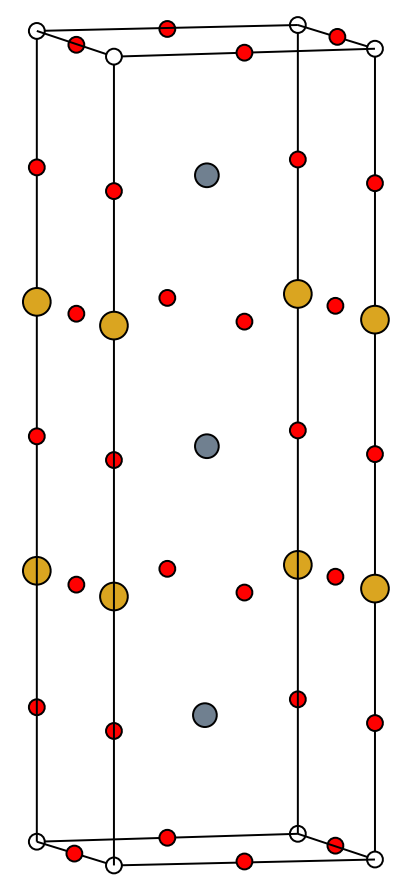

FIGURE 1. The [001]-ordered $\mathrm{Pb}\left(\mathrm{Zr}_{1 / 3} \mathrm{Ti}_{2 / 3}\right) \mathrm{O}_{3}$ supercell. Tables I and II give the corresponding atomic positions in the paraelectric and ferroelectric phases. 
The ferroelectric experimental ground state of $\mathrm{Pb}\left(\mathrm{Zr}_{1 / 3} \mathrm{Ti}_{2 / 3}\right) \mathrm{O}_{3}$ has the tetragonal $\mathrm{P} 4 \mathrm{~mm}$ point group and does not present any evidence of (long-range) B-site ordering. The material is thus composed of a succession of equivalent planes of composition $\mathrm{Zr}_{1 / 3} \mathrm{Ti}_{2 / 3}$ stacked along the tetragonal direction. To mimic this situation, we have chosen the ferroelectric direction in the supercell of Fig. 1 to lie along the [100] direction, rather than along the compositionally-modulated [001] direction. In our ferroelectric supercell, two axial ratios thus exist. These are the "ferroelectric-related" $a_{1} / a$ and the "ordered-related" $c / a$, where $a_{1}, a$, and $c$ are the lengths of the supercell lattice vectors along the [100], [010], and [001] directions, respectively. We are thus dealing with a P2mm orthorhombic ferroelectric supercell rather than with a P4mm tetragonal ferroelectric supercell. However, in order to be as close as possible to the experimental situation, we will keep the $c / a$ ratio as equal to the ideal value of 3 . In this case, we shall refer to our ferroelectric cell as "quasi-tetragonal" along [100] (i.e., tetragonal as regards the axial ratios, although true tetragonal symmetry is broken down to orthorhombic by the B-plane ordering in the [001] direction). The lattice parameter $a$, the axial ratio $a_{1} / a$, and the atomic positions along the [100] and [001] directions are then optimized in calculation (2) by minimizing the total energy and the Hellmann-Feynman forces (again to within a tolerance of $0.02 \mathrm{eV} / \AA$ for the forces).

The determination of the electronic ground state in calculations (1) and (2) is used to investigate the ferroelectric effects on the bond length distribution and on the chemical bonding in PZT with high Ti content. The effective charges in each case (i.e., in both non-centrosymmetric and centrosymmetric cells) will then be calculated from the polarization differences between the ground state and slightly distorted structures, following the procedure introduced in Ref. [8] and intensively used in Ref. [9].

\section{RESULTS}

\section{Centrosymmetric case}

Optimizing each degree of freedom in the centrosymmetric supercell leads to the lattice vectors $\vec{a}_{1, c}=a_{0}[1,0,0], \vec{a}_{2, c}=a_{0}[0,1,0]$, and $\vec{a}_{3, c}=a_{0}[0,0,2.99]$, where $a_{0}=7.498$ a.u. is the lattice parameter. The renormalized $c / a$ ratio defined as the actual ratio (i.e., 2.99) divided by the ideal one (i.e., 3.00) is equal to 0.997 and is thus very close to unity. For this reason, our centrosymmetric supercell can be referred as "quasi-cubic", which is consistent with the fact that the experimental paraelectric phase of PZT is cubic.

The relaxed atomic positions and the effective charges in this non-polar structure are given in Table I. It can be seen from Table I that (i) the $\mathrm{Pb}$ and $\mathrm{O}$ atoms lying between the $\mathrm{Zr}$ and $\mathrm{Ti}$ planes (i.e., $\mathrm{Pb} 1, \mathrm{O} 1, \mathrm{~Pb} 3$, and $\mathrm{O} 7$ ) move significantly towards the Ti planes; and (ii) the $\mathrm{Ti}$ and $\mathrm{O}$ atoms belonging to the Ti planes (i.e., Ti1, O2, O3, Ti2, O5, and O6) move very slightly towards the central mirror 
TABLE 1. Structural relaxations and effective charges for the [001] centrosymmetric supercell. The $\Delta z$ are the [001] atomic displacements of the non-polar structure with respect to the ideal ordered structure associated with $\vec{a}_{1, c}, \vec{a}_{2, c}$ and $\vec{a}_{3, c} . Z_{x x}$ and $Z_{z z}$ are the effective charges along the [100] and [001] direction, respectively.

\begin{tabular}{|c|c|c|c|c|c|c|}
\hline \multirow[b]{2}{*}{ Atoms } & \multicolumn{3}{|c|}{ Relaxed positions } & \multirow{2}{*}{$\begin{array}{c}\text { Displacements } \\
\quad \Delta z \text { (a.u.) }\end{array}$} & \multicolumn{2}{|c|}{ Effective charges } \\
\hline & $x$ (a.u.) & $y$ (a.u.) & $z$ (a.u.) & & $Z_{x x}$ & $Z_{z z}$ \\
\hline$\overline{\mathrm{Pb} 1}$ & 3.749 & 3.749 & -7.194 & 0.279 & 3.90 & 4.04 \\
\hline $\mathrm{Pb} 2$ & 3.749 & 3.749 & 0.000 & 0.000 & 3.88 & 3.53 \\
\hline $\mathrm{Pb} 3$ & 3.749 & 3.749 & 7.194 & -0.279 & 3.90 & 4.04 \\
\hline Ti1 & 0.000 & 0.000 & -3.643 & 0.093 & 6.77 & 6.65 \\
\hline Ti2 & 0.000 & 0.000 & 3.643 & -0.093 & 6.77 & 6.65 \\
\hline Zr1 & 0.000 & 0.000 & 11.210 & 0.000 & 6.33 & 6.69 \\
\hline O1 & 0.000 & 0.000 & -7.222 & 0.251 & -2.58 & -5.39 \\
\hline $\mathrm{O} 2$ & 3.749 & 0.000 & -3.638 & 0.099 & -5.58 & -2.34 \\
\hline O3 & 0.000 & 3.749 & -3.638 & 0.099 & -2.72 & -2.34 \\
\hline $\mathrm{O} 4$ & 0.000 & 0.000 & 0.000 & 0.000 & -2.53 & -5.57 \\
\hline O5 & 3.749 & 0.000 & 3.638 & -0.099 & -5.58 & -2.34 \\
\hline O6 & 0.000 & 3.749 & 3.638 & -0.099 & -2.72 & -2.34 \\
\hline $\mathrm{O} 7$ & 0.000 & 0.000 & 7.222 & -0.251 & -2.58 & -5.39 \\
\hline O8 & 3.749 & 0.000 & 11.210 & 0.000 & -5.17 & -2.94 \\
\hline O9 & 0.000 & 3.749 & 11.210 & 0.000 & -2.33 & -2.94 \\
\hline
\end{tabular}

$(\mathrm{PbO})$ plane. These atomic motions lead to shortened $\mathrm{Ti}-\mathrm{O}$ bonds and lengthened $\mathrm{Zr}-\mathrm{O}$ bonds. For example, the Ti1-O1 bond length shrinks to $1.89 \AA$, while Zr1-O7 enlarges to $2.11 \AA$, to be compared with the unrelaxed $\mathrm{B}-\mathrm{O}$ bond length of 1.98 $\AA$ in the ideal structure. As a matter of fact, the appearance of several different bond lengths associated with the mixed sublattice seems to be a general feature of alloying, and has also been observed and predicted in zinc-blende, wurtzite and rocksalt alloys [10-15].

Alloying effects in the present ordered [001] structure also lead to a change in the lengths of the $\mathrm{Pb}-\mathrm{O}$ bonds. For example, the $\mathrm{Pb} 3-\mathrm{O}$ bonds can be decomposed into three different groups: shorter Pb3-O bonds (e.g., Pb3-O5 equal to $2.73 \AA$ ), roughly unrelaxed $\mathrm{Pb} 3-\mathrm{O}$ bonds (e.g., $\mathrm{Pb} 3-\mathrm{O} 7$ equal to $2.80 \AA$ ), and long $\mathrm{Pb} 3-\mathrm{O}$ bonds (e.g., $\mathrm{Pb} 3-\mathrm{O} 8$ equal to $2.91 \AA$ ). The three groups are populated in the ratio 4:4:4. Thus, alloying has some significant effects on the B-O bonds $(\sim 4.5 \%$ change in bond lengths), and to a smaller extent, on the $\mathrm{Pb}-\mathrm{O}$ bonds ( $\sim 2.5 \%$ change).

The Born effective charges for our PZT supercell are detailed in Table I. They exhibit the same trends as in cubic bulk $\mathrm{PbTiO}_{3}$ and $\mathrm{PbZrO}_{3}$ compounds [9]: large values of about +4.0 for $\mathrm{Pb}$ atoms; large values around +6.5 for the $\mathrm{B}$ atoms; and two sets of values for the oxygen atoms, either close to -5.5 for oxygen atoms moving parallel to the $\mathrm{B}-\mathrm{O}-\mathrm{B}$ chain, or close to -2.5 for oxygen atoms moving perpendicular to these chains. The large values of the effective charges for $\mathrm{B}$ and $\mathrm{O}$ atoms are due to a (weak) hybridization between the $\mathrm{B} d$ and $\mathrm{O} 2 p$ orbitals 
TABLE 2. Structural relaxations and effective charges for the non-centrosymmetric supercell.

\begin{tabular}{|c|c|c|c|c|c|c|c|c|}
\hline \multirow[b]{2}{*}{ Atoms } & \multicolumn{3}{|c|}{ Relaxed positions (a.u.) } & \multicolumn{3}{|c|}{ Displacements (a.u.) } & \multicolumn{2}{|c|}{ Effective charges } \\
\hline & $x$ & $y$ & $z$ & $\Delta x$ & $\Delta y$ & $\Delta z$ & $Z_{x x}$ & $Z_{z z}$ \\
\hline $\mathrm{Pb} 1$ & 3.547 & 3.731 & -7.250 & -0.334 & 0.000 & 0.211 & 3.17 & 3.92 \\
\hline $\mathrm{Pb} 2$ & 3.551 & 3.731 & 0.000 & -0.331 & 0.000 & 0.000 & 3.37 & 3.56 \\
\hline $\mathrm{Pb} 3$ & 3.547 & 3.731 & 7.250 & -0.334 & 0.000 & -0.211 & 3.17 & 3.92 \\
\hline Ti1 & 0.015 & 0.000 & -3.630 & 0.015 & 0.000 & 0.101 & 5.38 & 5.81 \\
\hline $\mathrm{Ti} 2$ & 0.019 & 0.000 & 3.630 & 0.015 & 0.000 & -0.101 & 5.38 & 5.81 \\
\hline $\mathrm{Zr} 1$ & 0.118 & 0.000 & 11.192 & 0.118 & 0.000 & 0.000 & 6.06 & 6.06 \\
\hline O1 & 0.628 & 0.000 & -7.223 & 0.628 & 0.000 & 0.238 & -2.15 & -4.80 \\
\hline $\mathrm{O} 2$ & 4.432 & 0.000 & -3.637 & 0.551 & 0.000 & 0.094 & -4.56 & -1.95 \\
\hline O3 & 0.605 & 3.731 & -3.634 & 0.605 & 0.000 & 0.097 & -2.16 & -2.59 \\
\hline $\mathrm{O} 4$ & 0.656 & 0.000 & 0.000 & 0.656 & 0.000 & 0.000 & -2.10 & -4.92 \\
\hline $\mathrm{O} 5$ & 4.432 & 0.000 & 3.637 & 0.551 & 0.000 & -0.094 & -4.56 & -1.95 \\
\hline O6 & 0.605 & 3.731 & 3.634 & 0.605 & 0.000 & 0.097 & -2.16 & -2.59 \\
\hline $\mathrm{O} 7$ & 0.628 & 0.000 & 7.223 & 0.628 & 0.000 & -0.238 & -2.15 & -4.80 \\
\hline $\mathrm{O} 8$ & 4.201 & 0.000 & 11.192 & 0.320 & 0.000 & 0.000 & -4.62 & -2.63 \\
\hline O9 & 0.779 & 3.731 & 11.192 & 0.779 & 0.000 & 0.000 & -2.07 & -2.90 \\
\hline
\end{tabular}

$[9,16]$. It is interesting to note that along the [001] axis, the effective charge of $\mathrm{Ti}$ is very similar to that of $\mathrm{Zr}$, while the difference between these two effective charges in the bulk parent compounds is larger than 1.0 (i.e., 7.06 vs. 5.85 for $\mathrm{Ti}$ and $\mathrm{Zr}$ respectively, according to Ref. [9]). One can also point out that the effective charge along [001] for atom $\mathrm{O} 7$ sitting between the $\mathrm{Ti}$ and $\mathrm{Zr}$ atoms is -5.39, i.e., very close to the average value -5.32 of the corresponding oxygen effective charges in the bulk parents (-5.83 and -4.81 for $\mathrm{PbTiO}_{3}$ and $\mathrm{PbZrO}_{3}$ respectively, according to Ref. [9]).

\section{Ferroelectric effects}

We now turn to a consideration of the ferroelectric effects on bond-length distributions and effective charges. Optimizing each degree of freedom in the noncentrosymmetric cell previously described yields the following lattice vectors in atomic units: $\vec{a}_{1, n c}=a_{0}^{\prime}[1.040,0,0], \vec{a}_{2, n c}=a_{0}^{\prime}[0,1,0]$, and $\vec{a}_{3, n c}=a_{0}^{\prime}[0,0,3.00]$, where the lattice parameter is $a_{0}^{\prime}=7.461$ a.u., i.e., $0.5 \%$ smaller than for the centrosymmetric cell. A similar decrease of the lattice constant of around $0.7 \%$ has also been theoretically predicted when going from the paraelectric cubic phase to the tetragonal ferroelectric phase of the bulk $\mathrm{PbTiO}_{3}$ compound $[7,17]$. By looking at $\vec{a}_{1, n c}$, we also notice that our calculation predicts a "ferroelectric-related" axial ratio $a_{1} / a$ of 1.040. This prediction must be very close to the true value in $\mathrm{Pb}\left(\mathrm{Zr}_{1 / 3} \mathrm{Ti}_{2 / 3}\right)$, since recent measurements performed on $\mathrm{Pb}\left(\mathrm{Zr}_{1-x} \mathrm{Ti}_{x}\right)$ films [18] for $x=0.6$ found a value of 1.035 for this ratio, to be compared with values of 1.064 and 1.02 for $x=1$ and $x \simeq 0.5$ respectively [1]. The optimized non-centrosymmetric cell has 
an energy that is $0.15 \mathrm{eV} / 5$-atom-cell lower than that of the optimized centrosymmetric cell. This is consistent with the fact that the experimental ground state of $\mathrm{Pb}\left(\mathrm{Zr}_{1 / 3} \mathrm{Ti}_{2 / 3}\right)$ is ferroelectric and tetragonal rather than paraelectric and cubic. The relaxed atomic positions and the effective charges in the non-centrosymmetric structure are shown in Table II. The quantities $\Delta x, \Delta y$, and $\Delta z$ are the [100], [010] and [001] atomic displacements of the ferroelectric structure with respect to the ideal ordered structure associated with $\vec{a}_{1, n c}, \vec{a}_{2, n c}$ and $\vec{a}_{3, n c} . Z_{x x}$ and $Z_{z z}$ are the effective charges along the [100] and [001] directions, respectively.

A comparison of Tables I and II leads to the following observations. (i) The atomic displacements along the [001] direction are quite comparable between the centrosymmetric and the non-centrosymmetric phases. (ii) The ferroelectricity in tetragonal PZT is mainly characterized by the very large displacement of oxygen atoms along the tetragonal [100] direction (as in tetragonal $\mathrm{PbTiO}_{3}$ bulk), the large displacement of $\mathrm{Pb}$ atoms along the [100] direction, and by the slight displacement of Zr atoms along the [100] direction.

This ferroelectric atomic relaxation yields two different $\mathrm{Ti}-\mathrm{O}$ bond lengths along the [100] direction: a very long bond of length $2.33 \AA$, which is even longer than the longest $\mathrm{Zr}-\mathrm{O}$ bond $(2.16 \AA)$; and a very short bond of length $1.55 \AA$. This very short $\mathrm{Ti}-\mathrm{O}$ bond is much shorter than the shortest $\mathrm{Zr}-\mathrm{O}$ bond of $1.95 \AA$, and is even much shorter than the shortest $\mathrm{Ti}-\mathrm{O}$ bond of $1.78 \AA$ occurring in tetragonal ferroelectric $\mathrm{PbTiO}_{3}$.

Ferroelectricity also leads to a drastic change in the $\mathrm{Pb}-\mathrm{O}$ bonds. There are now some very short $\mathrm{Pb}-\mathrm{O}$ bonds with an average length of $2.51 \AA$, "normal" $\mathrm{Pb}-\mathrm{O}$ bonds with an average length of $2.84 \AA$, and very long $\mathrm{Pb}-\mathrm{O}$ bonds with an average length of $3.26 \AA$. As in the centrosymmetric cell, the population ratio between these three groups is again 4:4:4. However, the oxygens exhibiting the shortest $\mathrm{Pb}-\mathrm{O}$ bonds now share a common (100) plane, while they share a common (001) plane in the non-polar structure. Pair-distribution function analysis of recent pulsed neutron powder diffraction measurements on ferroelectric PZT alloys clearly confirms the existence of these three different groups [19]. The experimental average value of the three different $\mathrm{Pb}-\mathrm{O}$ bond lengths is $\sim 2.5 \AA, \sim 2.9 \AA$, and $\sim 3.4 \AA$, i.e., in excellent agreement with our predictions.

Comparing Table I and Table II also indicates that ferroelectricity leads to a striking decrease of the Born effective charges. The most spectacular decrease occurs for the atoms exhibiting a large change in their bond lengths. As a matter of fact, the effective charges along the [100] direction for atoms Ti1, Pb3 and O6 all decrease by $\sim 20 \%$ with respect to the centrosymmetric case. In consequence, the effective charges of the $\mathrm{Ti}$ atoms and $\mathrm{Pb}$ atoms are reduced by 1.4 and 0.7 , respectively, relative to their non-polar values. Previous theory has shown that a change of the effective charge by more than one unit of ' $e$ ' is indeed not unusual in going from the cubic to tetragonal ferroelectric phase in perovskite compounds $[20,21]$. The most extreme example appears to be for $\mathrm{Nb}$ in $\mathrm{KNbO}_{3}$, where the effective charges change from 9.67 to 7.05 in the direction parallel to the tetragonal axis. 

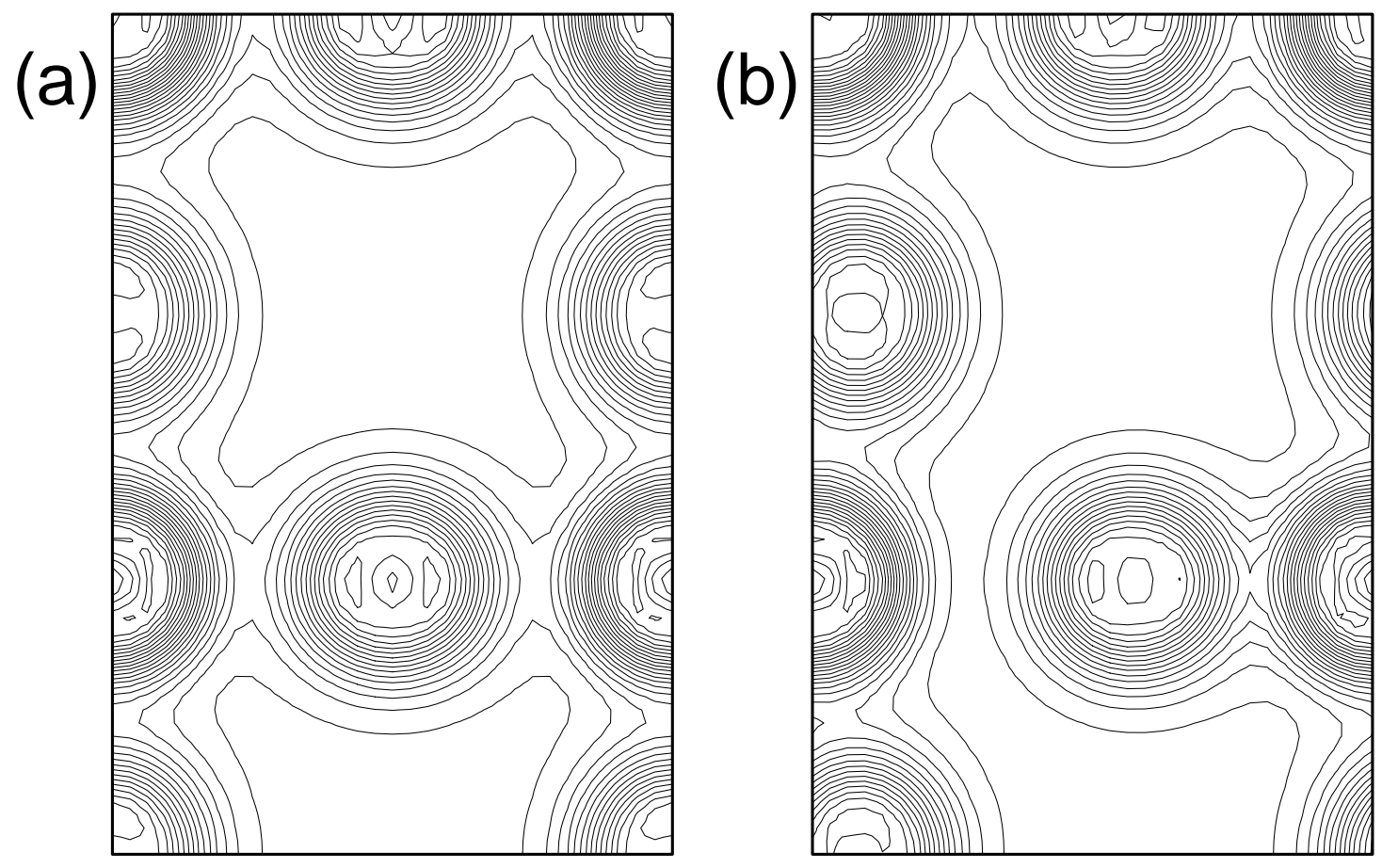

FIGURE 2. Electronic charge density plotted in the $(\mathrm{B}, \mathrm{O})$ plane for (a) paraelectric, and (b) ferroelectric, $\mathrm{Pb}\left(\mathrm{Zr}_{1 / 3} \mathrm{Ti}_{2 / 3}\right) \mathrm{O}_{3}$ supercells. Only the upper half of the supercell is shown; the horizontal and vertical axes lie along [100] and [001] respectively. The sequence of atoms appearing at the left and right edges is $\mathrm{Zr}$ (top), $\mathrm{O}, \mathrm{Ti}, \mathrm{O}$ (bottom); and the remaining middle atoms are oxygens.

To further understand the ferroelectric effects in PZT, Fig. 2 compares the electronic charge density in the $(\mathrm{B}, \mathrm{O})$ planes for the centrosymmetric and noncentrosymmetric cases. Figure 3 shows a similar comparison but in the $(\mathrm{Pb}, \mathrm{O})$ planes. Figure 2 indicates that ferroelectricity in PZT leads (i) to a chemical breaking of some $\mathrm{Ti}-\mathrm{O}$ bonds which generates the long $\mathrm{Ti}-\mathrm{O}$ bonds of $2.33 \AA$, and (ii) to the formation of strong covalency between $\mathrm{Ti}$ and $\mathrm{O}$, which is the cause of the very short $\mathrm{Ti}-\mathrm{O}$ bonds of $1.55 \AA$. In fact, we also found similar behavior for the electronic charge density in the $(\mathrm{Ti}, \mathrm{O})$ plane for cubic and tetragonal lead titanate (PT). Thus, as in bulk PT [22], the formation of ferroelectricity in tetragonal PZT leads to an enhancement of hybridization between Ti $3 d$ and $\mathrm{O} 2 p$ orbitals. Interestingly, we don't observe in Fig. 2 any breaking of $\mathrm{Zr}-\mathrm{O}$ bonds nor the formation of strong covalent $\mathrm{Zr}-\mathrm{O}$ bonds. The different chemical behavior between $\mathrm{Zr}$ and Ti may perhaps be the cause of the difference in ground states exhibited by the corresponding bulk parents (antiferroelectric and orthorhombic for $\mathrm{PbZrO}_{3}$ vs. ferroelectric and tetragonal for $\left.\mathrm{PbTiO}_{3}\right)$. The striking feature of Fig. 3 is the formation of covalent chains between $\mathrm{Pb}$ and $\mathrm{O}$ atoms, which is the cause of the 

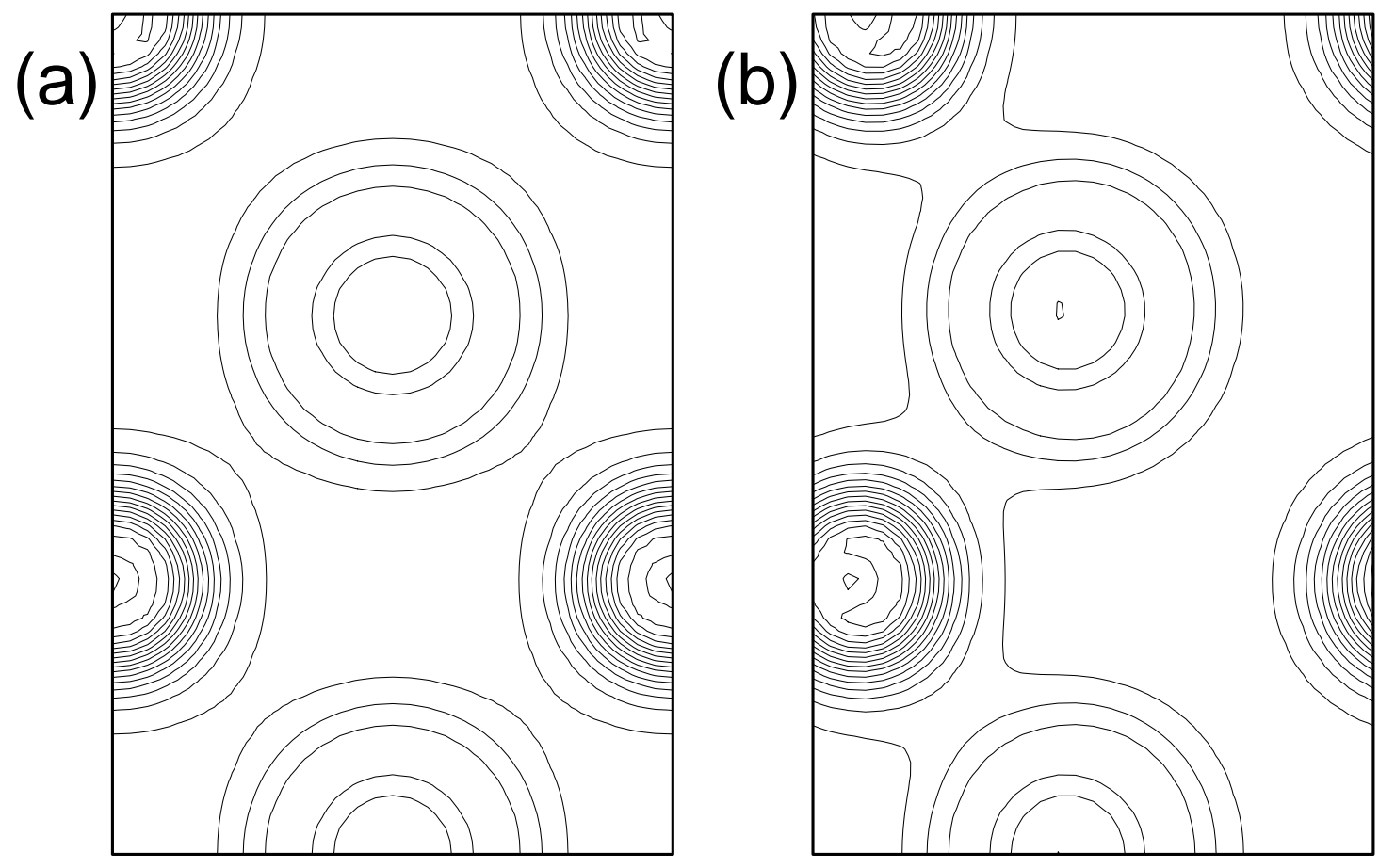

FIGURE 3. Electronic charge density plotted in the $(\mathrm{Pb}, \mathrm{O})$ plane for (a) paraelectric, and (b) ferroelectric, $\mathrm{Pb}\left(\mathrm{Zr}_{1 / 3} \mathrm{Ti}_{2 / 3}\right) \mathrm{O}_{3}$ supercells. Only the upper half of the supercell is shown; the horizontal and vertical axes lie along [100] and [001] respectively. Atoms appearing at the left and right edges are $\mathrm{O}$; atoms in the middle are $\mathrm{Pb}$.

very short $\mathrm{Pb}-\mathrm{O}$ bonds of $2.5 \AA$. We also found similar trends in the $(\mathrm{Pb}, \mathrm{O})$ planes of paraelectric and ferroelectric PT. Thus, as in bulk PT [22], the hybridization between $\mathrm{Pb} 6 s$ and $\mathrm{O} 2 p$ orbitals plays an important role in the ferroelectric behavior of tetragonal PZT.

\section{CONCLUSIONS}

Using 15-atom supercells and Vanderbilt ultrasoft pseudopotentials within the local-density approximation, we investigated alloying and ferroelectric effects on the bond lengths, chemical bonding and effective charges in lead zirconate titanate alloys (PZT).

Our principal findings are as follows.

(i) The centrosymmetric PZT alloy is mainly characterized by two sets of B-O bonds (shorter $\mathrm{Ti}-\mathrm{O}$ bonds vs. longer $\mathrm{Zr}-\mathrm{O}$ bonds), while the $\mathrm{Pb}-\mathrm{O}$ bonds differ only slightly (by $\sim 2.5 \%$ ) from the ideal structure.

(ii) Allowing ferroelectricity in PZT alloys has two striking chemical effects: enhancement of hybridization between $\mathrm{Ti} 3 d$ and $\mathrm{O} 2 p$ orbitals, and hybridization 
between $\mathrm{Pb} 6 s$ and $\mathrm{O} 2 p$ orbitals.

(iii) These chemical and ferroelectric effects lead to the formation of very short covalent $\mathrm{Ti}-\mathrm{O}$ bonds while breaking other $\mathrm{Ti}-\mathrm{O}$ bonds, and give rise to the formation of covalent chains of very short $\mathrm{Pb}-\mathrm{O}$ bonds.

(iv) The atoms engaged in covalent bonding exhibit a striking decrease of their effective charges by $\sim 20 \%$ relative to the paraelectric phase.

\section{ACKNOWLEDGMENTS}

This work is supported by the Office of Naval Research grant N00014-97-1-0048. We thank Professor T. Egami for helpful discussions and for communicating his results with us.

\section{REFERENCES}

1. M.E. Lines and A.M. Glass, Principles and Applications of Ferroelectrics and Related Materials (Clarendon Press, Oxford, 1977).

2. G. Saghi-Szabo and R.E. Cohen, Ferroelectrics 194, 287 (1997).

3. D. Vanderbilt, Phys. Rev. B 41, 7892 (1990).

4. D.M. Ceperley and B.J. Alder Phys. Rev. Lett. 45, 566 (1980).

5. J. Perdew and A. Zunger Phys. Rev. B 23, 5048 (1981).

6. H.J. Monkhorst and J.D. Pack, Phys. Rev. B 13, 5188 (1976).

7. R.D. King-Smith and D. Vanderbilt, Phys. Rev. B 49, 5828 (1994).

8. R.D. King-Smith and D. Vanderbilt, Phys. Rev. B 47, 1651 (1993).

9. W. Zhong, R.D. King-Smith and D. Vanderbilt, Phys. Rev. Lett. 72, 3618 (1994).

10. J.C. Mikkelsen and J.B. Boyce, Phys. Rev. Lett. 49, 1412 (1982).

11. Z. Wang and B.A. Bunker, Phys. Rev. B 46, 11277 (1992).

12. J.L. Martins and A. Zunger, Phys. Rev. B 30, 6217 (1984).

13. N. Marzari, S. de Gironcoli and S. Baroni, Phys. Rev. Lett. 72, 4001 (1994).

14. C.K. Shih, W.E. Spicer, W.A. Harrison, and A. Sher, Phys. Rev. B 31, 1139 (1985).

15. L. Bellaiche, S.H. Wei and A. Zunger Phys. Rev. B 56, 13872 (1997).

16. M. Posternak, R. Resta and A. Baldereschi, Phys. Rev. B 50, 8911 (1994).

17. A. Garcia and D. Vanderbilt, Phys. Rev. B 54, 3817 (1996).

18. W. Zhu, Z.Q. Liu, W. Lu, M.S. Tse, H.S. Tan and X. Yao, J. Appl. Phys. 79, 4283 (1996).

19. T. Egami, S. Teslic, W. Domowski, P.K. Davies and I.-W. Chen, submitted (1997).

20. Ph. Ghosez, X. Gonze, Ph. Lambin and J.-P. Michenaud Phys. Rev. B 51, 6765 (1995).

21. C.-Z. Wang, R. Yu and H. Krakauer, Phys. Rev. B 54, 11161 (1996).

22. R.E. Cohen, Nature 358, 136 (1992). 